JASMín HerRera de LA BARRERA

Magíster en Ingeniería Industrial por la Universidad Simón

Bolívar

Docente de la Fundación Universitaria Tecnológico Comfenalco - Cartagena

Colombia

[Jherrerad@tecnocomfenalco.edu.co]

Juan Miguel Martínez Buendía Magíster en Ciencias Estadísticas por la Universidad Nacional de Colombia Investigadora vinculada a la Escuela Naval de Cadetes Almirante Padilla Colombia

[aramisdegaula1977@gmail.com]

LAURA Martínez García Magíster en Ingeniería de Sistemas por la Universidad Tecnológica de Bolívar Docente de la Fundación Universitaria Tecnológico Comfenalco - Cartagena Colombia [Imartinezg@tecnologicocomfenalco.edu.co]

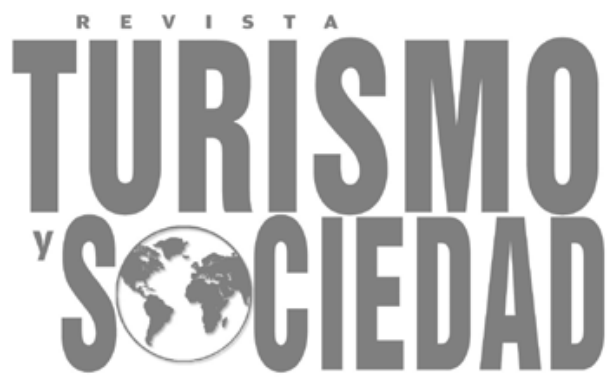

\section{SOFTWARE DE SOSTENIBILIDAD TURÍSTICA PARA EL CUMPLIMIENTO DE LA NTS COLOMBIANA ${ }^{1}$}

\section{TOURISM SUSTAINABILITY SOFTWARE FOR COMPLIANCE WITH THE COLOMBIAN NTS}

\footnotetext{
1 Para citar el artículo: Herrera, J., Martínez, J. y Martínez. A. (2021). Software de sostenibilidad turística para el cumplimiento de la NTS colombiana. Turismo y Sociedad, XXVIII, pp. 187-205. DOI: https://doi.org/10.18601/01207555.n28.09

Fecha de recepción: 17 de julio de 2019

Fecha de modificación: 27 de septiembre de 2019

Fecha de aceptación: 2 de diciembre de 2019
}

\section{Resumen}

Este artículo se basa en el diseño, el desarrollo y la evaluación de una herramienta tecnológica llamada "NTSOFT" como instrumento de utilidad empresarial para prestadores de servicios turísticos, que permita generar un diagnóstico y estadísticas que identifiquen el nivel de cumplimiento de los establecimientos ante los criterios normativos de calidad de la norma técnica sectorial colombiana. En el proyecto se utilizaron dos metodologías para el desarrollo de sistemas de información que dieron como resultado la construcción de un marco conceptual del tema, un diagnóstico del problema y un análisis para identificar y definir los objetivos del software por medio de un modelo para la implementación de la norma, así como el diseño y desarrollo del sistema y su evaluación a partir de una muestra representativa de 37 expertos, para lo cual se empleó la técnica de índice de concordancia. Los resultados de esta evaluación van dirigidos a validar un uso óptimo de los recursos ambientales, que son un elemento fundamental del desarrollo turístico, y al aseguramiento de las actividades económicas a largo plazo que garanticen el cumplimiento de la norma técnica sectorial impuesta por el Gobierno colombiano.

Palabras claves: Norma técnica sectorial, sostenibilidad, calidad, turismo, software.

\section{Abstract}

The growth of the tourism sector is becoming increasingly important in terms of competitiveness and sustainability of tourism destinations. For this reason, this paper presents the design, development and evaluation of tourism sustainability software as a useful business tool for service providers. The software called "NTSOFT", allows you to generate a diagnosis and statistics that identify the level of compliance of 
the tourist establishments to the normative quality criteria of the Technical Provisions of the Sector. In this way, mechanisms are put into practice to evaluate the ongoing improvement, control and measurement of the sector through management indicators that guarantee establishment designs and that monitor their action plans. All this designed to provide the evidence required for the procedures at the registry of tourism.

Keywords: Sectoral technical standard, sustainability, quality, tourism, software.

\section{Introducción}

Para garantizar los niveles de competitividad en turismo, se requiere de una eficiente gestión del destino y de los prestadores de servicios, con el fin de minimizar los impactos ambientales, socioculturales y económicos que resultan de la actividad (Trujillo-Casañola, Febles Estrada y León-Rodríguez, 2014). Actualmente, los establecimientos prestadores de servicios turísticos enfocan sus esfuerzos en lograr el reconocimiento y alcanzar el equilibrio entre la sostenibilidad y la actividad económica. Sin embargo, esto no es suficiente para llegar a convertirse en establecimientos de "turismo sustentable", ya que es necesario que los productos y servicios ofrecidos estén acompañados de instrumentos y normas de gestión que garanticen su calidad.

El Ministerio de Comercio, Industria y Turismo de Colombia (MinciT), el Icontec ${ }^{2}$, la academia, los gremios y prestadores de servicios turísticos del país conforman la Unidad Sectorial de Normalización en

2 El Instituto Colombiano de Normas Técnicas y Certificación (Icontec) es el organismo nacional de normalización, según el Decreto 2269 de 1993 del Ministerio de Desarrollo Económico (hoy Ministerio de Comercio, Industria y Turismo), y delega el proceso de elaboración de normas técnicas a cada sector que lo requiera y determine sus necesidades.
Sostenibilidad Turística — tanto para destinos turísticos como para prestadores de servicios - con el fin de proponer normas que promuevan prácticas sostenibles (Instituto Colombiano de Normas Técnicas y Certificación (Icontec), 2014). El objetivo de esta Unidad es estandarizar los criterios para la implementación de sistemas de gestión para la sostenibilidad que permitan a los establecimientos minimizar los impactos ambientales, socioculturales y económicos de su actividad económica en el entorno en el cual operan (MinciT, 2015a).

La iniciativa consiste en crear una cultura de excelencia en la prestación de servicios turísticos a los consumidores por medio de un certificado de calidad turística. Con esta iniciativa, el Gobierno nacional, a partir del Plan Sectorial de Turismo 2014-2018 "Turismo para la construcción de la paz", busca posicionar a Colombia como destino turístico sostenible y sustentable, así como adquirir un reconocimiento a nivel mundial $\mathrm{y}$ fortalecer el mercado interno mediante políticas sectoriales que permitan mejorar los niveles de competitividad e incentivar estrategias de promoción con participación del sector privado (MinCIT-DNP, 2014).

En Colombia existen 57 normas técnicas sectoriales asociadas con la calidad turística de los establecimientos prestadores de servicios (EPS), sin embargo, la medida de la adopción normativa no había sido eficaz. Por esta razón, a partir de la Resolución 3860 de 2015 (MincIT, 2015b) se reglamenta que los prestadores de servicios turísticos que dispongan de normas técnicas sectoriales relacionadas con la sostenibilidad turística deben actualizar el Registro Nacional de Turismo (RNT) y demostrar las obligaciones exigidas y el cumplimiento de los requisitos contemplados en la norma que les corresponda (Muñoz Gómez, 2017). Esta norma motiva a diseñar alternativas para su implementación; una de ellas es el presente 
trabajo de investigación, que mediante el desarrollo de una herramienta tecnológica busca soportar el esquema documental de los establecimientos de turismo para garantizar la trazabilidad de las actividades, evaluar la mejora continua, facilitar el control y la medición por medio de indicadores de gestión y realizar seguimiento de los planes de acción implementados. A su vez, esta herramienta facilita a los entes de control y auditoría la verificación de los criterios normativos establecidos y proporciona la evidencia requerida para los trámites en lo concerniente al Registro Nacional de Turismo.

El documento se encuentra organizado de la siguiente manera: en la segunda sección se describe la metodología, en la tercera parte se exponen los resultados y se finaliza con las conclusiones.

\section{Metodología}

La metodología del proyecto está soportada en la propuesta de Nunamaker y Chen (1990) titulada "Systems development in information systems research", un enfoque metodológico para la investigación orientado al desarrollo de sistemas a partir de cuatro estrategias: desarrollo de teoría, experimentación, observación y desarrollo del sistema. También se tomó como base el enfoque denominado "Multi-methodological approaches in design science", de Bai, White y Sundaram (2013), quienes afirman que el desarrollo de sistemas y otras metodologías de investigación son complementarios y que un enfoque integrado multidimensional y multimetodológico generará resultados fructíferos. La premisa es que las contribuciones en investigación pueden resultar del desarrollo de sistemas, de la experimentación, la observación y de pruebas de desempeño, y que todos estos enfoques de investigación son necesarios en los diferentes aspectos de la pregunta de investigación.
A continuación, se presenta el proceso de desarrollo del sistema propuesto desde una perspectiva metodológica en investigación, en el que se tuvieron en cuenta cuatro fases para el desarrollo del proyecto:

Fase 1. Construir un marco conceptual del tema de investigación en sostenibilidad turística, norma sectorial y trabajos relacionados con la temática.

Fase 2. Realizar un diagnóstico del problema y un análisis que permitan identificar y definir los objetivos del software en sostenibilidad turística a partir de un modelo para la implementación de la norma técnica sectorial en los establecimientos prestadores de servicios turísticos.

Fase 3. Diseñar y construir el sistema para validar la teoría propuesta y los diseños del sistema por medio de una arquitectura de software. A partir de este análisis, se diseña un modelo que constituye la base conceptual del prototipo que se desarrolla como apoyo al sistema.

Fase 4. Evaluar el sistema a partir de una muestra representativa de 37 expertos mediante la técnica del índice de concordancia. Los resultados de esta evaluación van dirigidos a validar un uso óptimo de los recursos ambientales, que son un elemento fundamental del desarrollo turístico, y al aseguramiento de las actividades económicas a largo plazo que garanticen el cumplimiento de la norma técnica sectorial impuesta por el Gobierno colombiano.

\section{Marco conceptual}

\subsection{Turismo sostenible}

La Organización de las Naciones Unidas para la Educación, la Ciencia y la Cultura (Unesco, 2012) define la sostenibilidad como "un paradigma para pensar en un futuro en 
el cual las consideraciones ambientales, sociales y económicas se equilibran en la búsqueda del desarrollo y de una mejor calidad de vida". La sostenibilidad se considera como un objetivo alcanzable por medio del desarrollo sostenible, el cual es aquel que cumple las necesidades de hoy sin degradar las condiciones con las que las próximas generaciones convivirán. De allí que el turismo sostenible comprende los aspectos ambientales, económicos y socioculturales en el desarrollo de la actividad turística para garantizar su sostenibilidad a largo plazo (Arguello, Fiallos, Chinchuela, Díaz, Verdugo, Jara y Quezada, 2017).

La OMT define el turismo sostenible así:

Aquel que busca una gestión de recursos que satisfaga todas las necesidades económicas, sociales y estéticas de la empresa, y a la vez respete la integridad cultural, los procesos ecológicos esenciales, la diversidad biológica y los sistemas de soporte de la vida.

Por su parte, Beatriz Navarrete Cobo (2011) considera que algunos aspectos para llegar al turismo sostenible son eliminar los problemas de ordenación territorial, equilibrar la explotación turística, tener en cuenta la tendencia demográfica de la población actual, preservar la situación competitiva del sector turístico y crear un nuevo modelo de turismo sostenible.

Al conceptualizar las dimensiones de la sostenibilidad, Catalano (2013) define la dimensión sociocultural como una reacción dependiente del estilo de vida de las personas, el cual, junto con la actividad económica de los residentes, es un factor decisivo a la hora de determinar su destino turístico. Suele suceder que la diversidad cultural y las actividades rutinarias pueden impactar en el atractivo medioambiental del destino.
De acuerdo con el contenido del análisis sobre turismo sostenible para el desarrollo realizado en el año 2017 por la UNWTO, el sector turístico contribuye en los tres pilares de la sostenibilidad: medioambiental, económico y social. Por lo tanto, se sugiere el uso de instrumentos útiles para alcanzar el cumplimiento de los Objetivos para el Desarrollo Sostenible - algunos de estos orientados al uso de la tierra-, alternativas financieras e incentivos fiscales, sistemas de certificación y buenas prácticas (UNWTO, 2017).

Otras corrientes consideran la sostenibilidad turística como un saber transversal a varias disciplinas y que cobra importancia dado el impacto que tiene el sector hotelero sobre la gestión ambiental y la economía del destino. En este caso se proponen metodologías para la medida del costo para la sostenibilidad ambiental y certificaciones como ayuda para la medida de la eficiencia y la gestión ambiental en la industria hotelera (Da Rosa y Silva, 2017).

La sustentabilidad resulta la justificación perfecta para diversificar la oferta turística vigente, tomando en cuenta las variables ambiental, social y cultural como una fortaleza, no como una amenaza, así se logró crear conciencia acerca de la necesidad de cuidar los recursos tanto naturales como culturales y contar con una nueva ola de turistas más conscientes con el medioambiente (Sánchez Valdés y Vargas Martínez, 2015). Sin embargo, en Colombia, algunas prácticas turísticas han presentado algunos inconvenientes: la baja calidad ambiental; la contaminación de aguas de baño; el retroceso de la línea de costa como resultado de las dinámicas físiconaturales; el déficit en el ordenamiento y la planificación de las playas; la ocupación ilegal de las zonas de playa por construcciones inmobiliarias, infraestructura, invasiones y demás usos no autorizados en bienes de uso público (Zúñiga-Collazos, 2015). Con respecto a estas situaciones, el Gobierno 
nacional ha fomentado la implementación de las normas técnicas sectoriales, orientadas al cumplimiento normativo para minimizar los aspectos ambientales, socioculturales y económicos que resulten significativos en el destino donde se desarrollan las actividades turísticas (MinciT, 2015).

\subsection{Norma Técnica Sectorial TS 002}

En el año 1996, el Gobierno nacional emitió la Ley 300 (Ley General de Turismo) (Congreso de Colombia, 1996), por medio de la cual se busca el mejoramiento de la calidad de los servicios turísticos prestados y la creación de Unidades Sectoriales. De igual forma, el Ministerio de Comercio, Industria y Turismo, con apoyo de la Universidad Externado de Colombia, creó en 2004 la Unidad Sectorial de Normalización en Sostenibilidad Turística con el fin de incentivar los procesos de calidad en la prestación de servicios turísticos; para ello se publicó el compendio de normas técnicas sectoriales (en total 52) asociadas con cada uno de los prestadores de servicios turísticos y destinos, entre las cuales está la NTS TS 002: Establecimientos de alojamiento y hospedaje. Requisitos de sostenibilidad turística (Icontec, 2014).

En el año 2012, el Gobierno nacional publicó la Ley 1558 (Congreso de Colombia, 2014), cuyo artículo $5 .^{\circ}$ se refiere a la calidad turística. Esta ley dicta que las NTS son de estricto cumplimiento para todos los prestadores de servicios turísticos, de acuerdo con la reglamentación que expide el Ministerio de Comercio, Industria y Turismo.

En 2014, con la Resolución 2804 (MinciT, 2014) se reglamentó el cumplimiento de las normas técnicas de calidad expedidas por las Unidades Sectoriales de Normalización sobre Sostenibilidad Turística. La Resolución establece que los prestadores de servicios turísticos que dispongan de normas técnicas sectoriales relacionadas con la sostenibilidad turística deberán cumplir las normas vigentes, sus actualizaciones y las que se lleguen a expedir según la actividad que les corresponda.

La NTS 002:2014 (Icontec, 2014) es una norma técnica compuesta por siete capítulos básicamente, pero los que presentan obligatoriedades son el capítulo 3 (gestión para la sostenibilidad), el 4 (requisitos ambientales), el 5 (requisitos socioculturales) y el 6 (requisitos económicos). Los establecimientos de alojamiento y hospedaje que deseen demostrar conformidad con esta norma técnica deberán cumplir la totalidad de los requisitos, buscar el equilibrio entre cada una de las dimensiones de la sostenibilidad, así como identificar y mitigar aquellos aspectos ambientales, culturales y socioeconómicos significativos generados por la actividad turística.

\subsection{Trabajos relacionados en el desarrollo de sistemas con base en la NTS}

En el mundo son muchos los países que han desarrollado metodologías para la aplicación voluntaria de la sostenibilidad en los establecimientos prestadores de servicios turísticos; países como España y México han logrado un impacto positivo del desempeño ambiental estableciendo prácticas apropiadas (Llull Gilet, 2001; Pardo, 2017). Así mismo, hay trabajos relacionados con el tema, como es el caso de la investigación de Cerezo y Guevara (2015) sobre la importancia de los planes estratégicos y de las TIC en el turismo. Estos autores proponen realizar un estudio prospectivo en el interior de las organizaciones que propenda al acompañamiento de la actividad turística con el desarrollo de plataformas que permitan la visibilidad y el control de sus productos.

Las TIC y el turismo han crecido en forma paralela y se han convertido en elementos esenciales en la globalización. Ambos han 
generado un complemento que ayuda al impulso del sector turístico por medio de la difusión de productos y dando a conocer los ambientes propicios para el descanso (Cerezo y Guevara, 2015).

A partir de este concepto, se ha generado una sensibilización hacia el tema de la sostenibilidad, en el que participa toda la cadena de valor del sector turismo en lo referente a la elaboración e implementación de estrategias para el cuidado del medioambiente, de los aspectos culturales y también de los económicos. Si el desarrollo turístico genera beneficios, estos deben propender a la conservación de los recursos naturales y culturales en los que se basa la prosperidad del ser humano (Catalano, 2013). Un concepto similar al de sostenibilidad es el de responsabilidad social empresarial (RSE), que se fundamenta en la creación de valor para los grupos de interés y busca un equilibrio entre ellos y el ambiente en el que se desarrolla la actividad (Barquero, 2017).

Por su parte, el Gobierno colombiano ha promovido políticas encaminadas a mantener el equilibrio económico del sector y su impacto en el medio ambiente (Ochoa y Rueda, 2017). Entre los proyectos que han implementado la norma técnica sectorial en turismo sostenible en Colombia se encuentra el de Roa Rodríguez y Sánchez Giraldo (2019), que plantea un modelo de gestión que establece el método para medir el desempeño de variables de acuerdo con la NTS-TS-002; dicho modelo asegura demostrar avances en la mejora continua de los requisitos de sostenibilidad por ella exigidos. Por su parte, Caviedes y Olaya (2018) presentan una revisión de impactos ambientales con énfasis en las normas técnicas de sostenibilidad ambiental en áreas protegidas de Colombia con vocación ecoturística. De igual manera, el grupo de investigación Turismo y Sociedad de la Universidad Externado de Colombia (Ochoa y Rueda, 2017) analizó los efectos de la implementación de la NTS en los prestadores de servicios turísticos y en los destinos que se encontraban certificados en alguna de las normas técnicas realizadas hasta la fecha del estudio. Este trabajo se centró en entender cómo las empresas y los destinos ven la certificación en turismo sostenible y qué deben revisar, entender y analizar de los requisitos definidos en la norma para poder cumplirlos.

Por último, con base en estas necesidades presentes en el sector turístico, se desarrolló un proyecto de investigación que propone un modelo de gestión del estándar de la NTS-TS 002:14 por medio de una aplicación web para admitir el proceso de diagnóstico y seguimiento de los indicadores incluidos en la norma (García, Herrera y Puello, 2018).

\section{Diagnóstico y análisis del contexto problema}

La segunda fase del proyecto corresponde al diagnóstico realizado a los afiliados a Cotelco Bolívar en el año 2016 por medio de un instrumento que contempla los criterios fijados en la NTS 002:2014 (Icontec, 2014). Los ítems fueron formulados de acuerdo con una escala de Likert y permiten determinar el grado de cumplimiento de la norma por parte de los establecimientos que participaron en el estudio. Con este instrumento se pretende recopilar información por medio de la cual se pueda definir un modelo para la implementación de la norma técnica sectorial en los establecimientos prestadores de servicios turísticos.

El instrumento fue validado a partir del Alpha de Cronbach con un valor de 0,9813. La información procesada muestra que el $60 \%$ de los locales diagnosticados implementan de manera empírica algunos de los requisitos, pero no guardan registros que evidencien el desempeño de los procesos. De lo anterior se infiere que los establecimientos 
Figura 1. Cumplimiento por requisitos

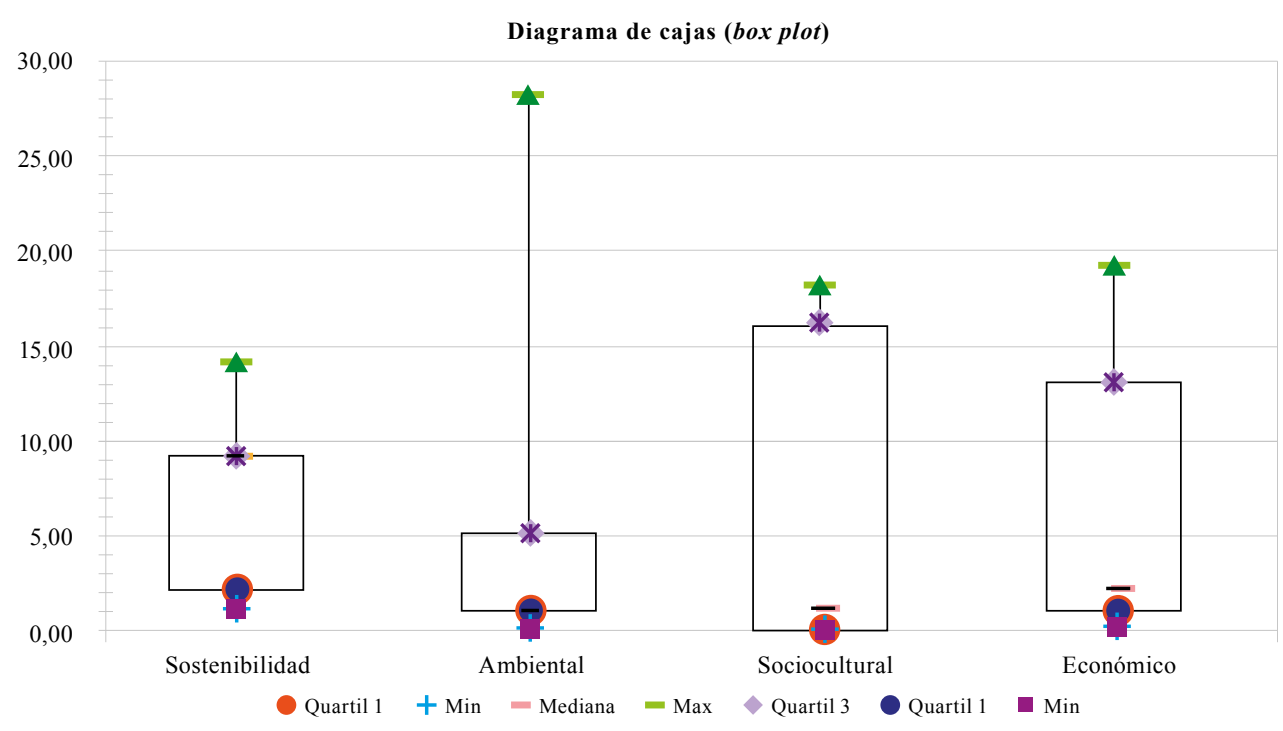

Fuente: Elaboración propia.

tienen conciencia de la importancia de las dimensiones ambientales, socioculturales y económicas, sin embargo, no aplican la norma. El $34 \%$ de los establecimientos presentan falencias relacionadas con operaciones que no son realizadas en su interior, pero que son de carácter obligatorio. Solo un $6 \%$ de los locales presentan un adelanto en la implementación y cumplen tanto con la documentación mínima requerida como con la coherencia en la aplicación de la norma.

Consecuentemente, se realizó un análisis del comportamiento de las dimensiones de la sostenibilidad (Aguiar y López, 2017) con el fin de establecer la tendencia de cumplimiento de los establecimientos. Al respecto, se determinó que el componente ambiental es aquel cuya implementación resulta más difícil, seguido por los requisitos de sostenibilidad, dada la complejidad de las actividades propuestas por la norma. Por su parte, la gestión de los establecimientos hacia las dimensiones sociocultural y económica tiene comportamientos similares y de fácil entendimiento por el vínculo cerrado que existe entre las actividades de turismo, la riqueza patrimonial de la ciudad y el impulso económico de esta actividad sobre la región. Lo anterior se ilustra en la figura 1 .

\section{Diseño y desarrollo del sistema NTSOFT}

\subsection{Modelo conceptual}

Como lo demuestran los resultados del diagnóstico, se hace necesario el diseño de un modelo para la implementación de la norma técnica sectorial que permita a los establecimientos prestadores de servicios turísticos el cumplimiento normativo, así como analizar, controlar, hacer seguimiento y mejorar la gestión de sus procesos en pro del desarrollo sostenible.

La norma técnica sectorial opera como una lista de chequeo; no se basa en los principios básicos del enfoque sistémico, ya que no orienta la gestión de los procesos. Teniendo en cuenta lo anterior, se realiza un análisis estructural con expertos del sector en el que 
se definen las variables que forman parte del modelo y la secuencia de las actividades de acuerdo con el ciclo PHVA (planear, hacer, verificar y actuar) (Ballesteros, Bohórquez, Delgado, Pérez y Pinzón, 2017).

El modelo propuesto integra la filosofía de la calidad total, el modelo Iso y el desarrollo sostenible. El modelo está constituido por variables influyentes que determinan los elementos de entrada al sistema de gestión sobre los cuales se adecuan las actividades turísticas del establecimiento a partir de las dimensiones de la sostenibilidad, teniendo en cuenta que entre los principios de la planeación se encuentran la determinación de las capacidades y la asignación de los recursos necesarios para garantizar el éxito operacional.

Se incorpora un componente denominado liderazgo, proveniente de la filosofía de la calidad total y del modelo ISO, y como variables dependientes se encuentran la operación y el resultado, las cuales constituyen el hacer del establecimiento bajo parámetros de desarrollo sostenible. El enfoque hacia el resultado es la entrada a la mejora continua, orientada hacia la satisfacción de los grupos de interés y al crecimiento de la organización. En el modelo propuesto, la revisión por la dirección deja de ser una actividad de control y se convierte en una actividad de gestión que opera tanto en el análisis de los datos como en la toma de decisiones basada en hechos y en las relaciones mutuamente beneficiosas con el entorno (figura 2).

A partir de este análisis, se generan estrategias para la implementación de un software que se adapte al modelo propuesto, permita la dinamización de cada uno de los componentes y facilite el entendimiento del modelo en los establecimientos de alojamiento y hospedaje.
Figura 2. Modelo propuesto

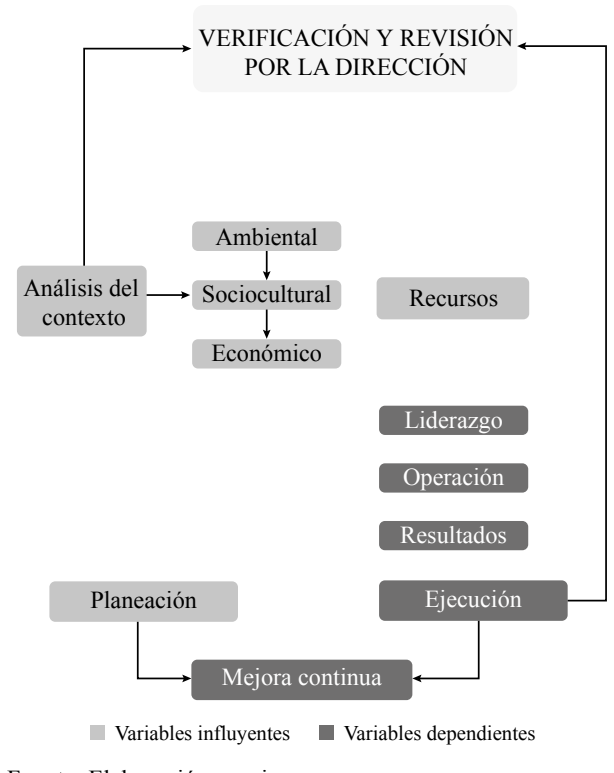

Fuente: Elaboración propia.

\subsection{Diseño y arquitectura del sistema}

La implementación del modelo hace necesario que la organización cuente con mecanismos eficientes para el seguimiento y control del entorno de sus actividades económicas, esto con el fin de adaptarse a los cambios mediante un ejercicio flexible y respetando su estructura y pertinencia con respecto a la norma técnica. Esta herramienta tecnológica se centra en la minimización de los impactos significativos generados desde las dimensiones ambiental, sociocultural y económica que emprende el establecimiento en el desarrollo de sus actividades turísticas.

El diseño del sistema NTSOFT se desarrolló por medio de la arquitectura empresarial Java Enterprise Edition como una aplicación web bajo el modelo N-Capas con el fin de hacerla extensible y fácil de mantener en el tiempo. Además, la arquitectura tomó la visión del trabajo presentado por los autores Martínez, Contreras, Mendoza y Torres (2015) en lo 
referente al uso de tecnologías, comunicación entre componentes y patrón de diseño.

El sistema fue implementado bajo el framework Spring MVC, que permite crear código de alto rendimiento, liviano y reutilizable, con la finalidad de estandarizar, agilizar, manejar y resolver los problemas que puedan ir surgiendo en el desarrollo de la programación del proyecto. Spring ofrece soporte de infraestructura en lo concerniente a la aplicación y brinda un completo modelo tanto para la configuración como para la programación de aplicaciones empresariales desarrolladas bajo Java.
Las capas que componen el sistema en su primera versión son tres: presentación, negocio e integración. La capa de presentación "representa la interfaz de usuario que maneja el cliente a través del navegador", la cual simplifica el desarrollo de las interfaces de usuario, instanciación y disponibilidad al cliente por medio de las páginas JSP. Por su parte, la capa de negocio "contiene los componentes de negocio [...] que automatizan los procesos que llevan a cabo los usuarios" por medio de un "conjunto de servicios web y objetos de negocio" (Martínez, Mendoza y Puello, 2016, p. 69). Los servicios web implementados tienen diversas funcionalidades,

Figura 3. Arquitectura del sistema NTSOFT
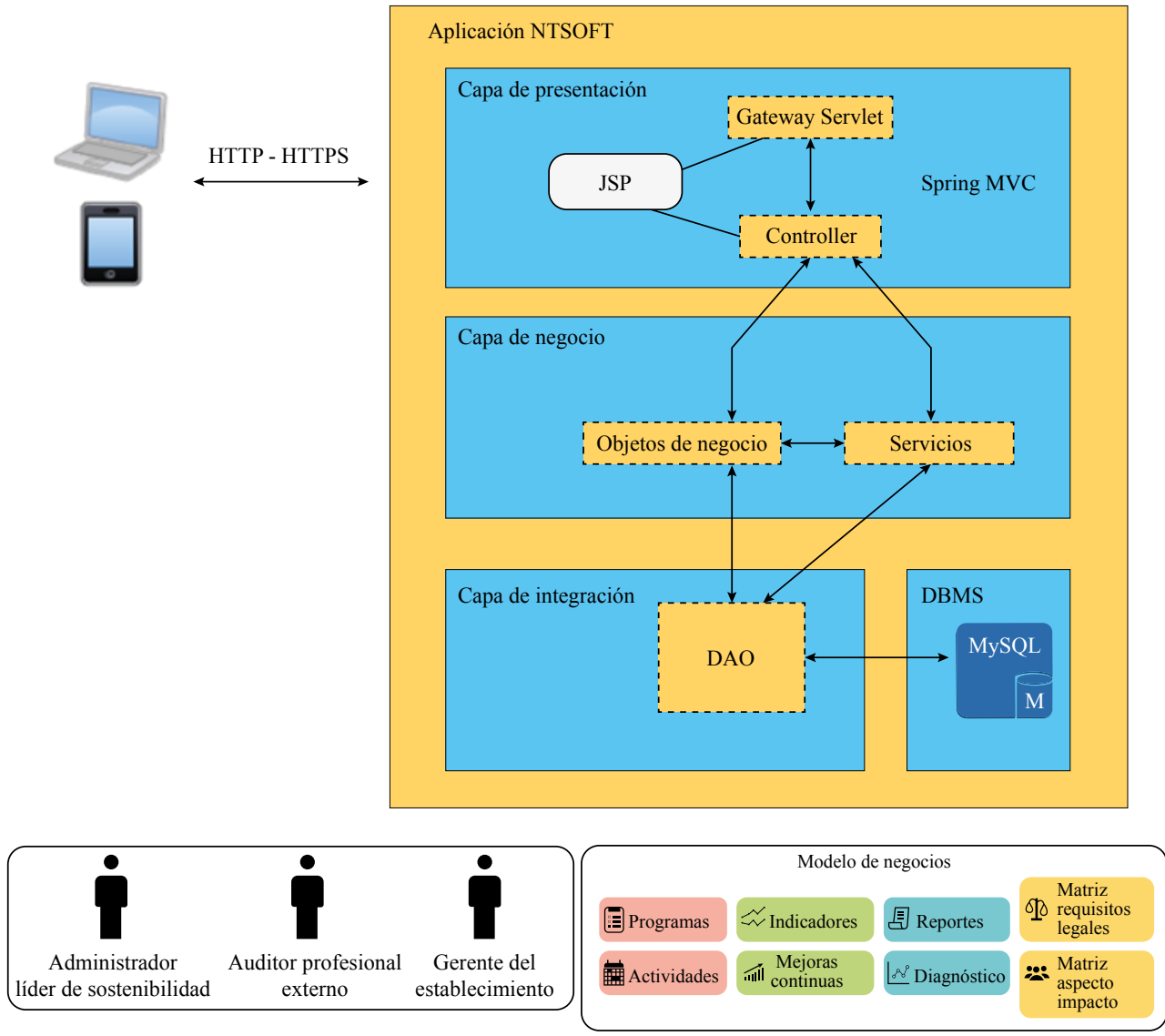

Fuente: Elaboración propia. 
por ejemplo, el cumplimiento normativo con relación a la NTS; el diseño de programas de sostenibilidad ambiental, sociocultural y económica; y el mejoramiento continuo en indicadores de eficiencia, indicadores de efectividad y generación de reportes para la toma de decisiones. Finalmente, la capa de integración permite la unión entre la implementación del aplicativo y cualquier otro sistema de la organización. "Los datos compartidos entre los componentes de negocio y las invocaciones de persistencia de la aplicación se mantienen en una base de datos MysQL" (Martínez et al., 2016, p. 70) por medio de la tecnología DAO (objetos de acceso a datos), con el fin de aislar la aplicación de la tecnología subyacente con la que fue construida.

\subsection{Prototipo: plataforma NTS-SOFT}

Una vez definidos el modelo de implementación y la arquitectura del sistema, se procedió al desarrollo del prototipo, el cual cuenta con las utilidades que se muestran en la figura 4 .

Se presentan a continuación cada uno de los módulos que contiene el sistema.

\subsubsection{Módulo de dispositivo normativo}

Este módulo, denominado "Diagnóstico" en la plataforma, permite determinar el estado de cumplimiento del establecimiento de alojamiento y hospedaje en relación con los requisitos exigidos por la NTS TS 002: 2014 en lo que concierne a las dimensiones ambiental, sociocultural y económica. Por tanto, los requisitos son evaluados por medio de una escala de 0 a $100 \%$, que varía de acuerdo con el estado de cumplimiento del requisito o con su evidencia de soporte. Las escalas son:

- No cumple

- No está documentado

- No hay evidencia de cumplimiento

- Documentado, pero no implementado

- Cumplimiento total.

Los resultados del diagnóstico serán tomados como referencia inicial para que el responsable del sistema de gestión determine las debilidades que debe mejorar y las fortalezas que ha de mantener para dar cumplimiento

Figura 4. Funcionalidades del sistema NTSOFT

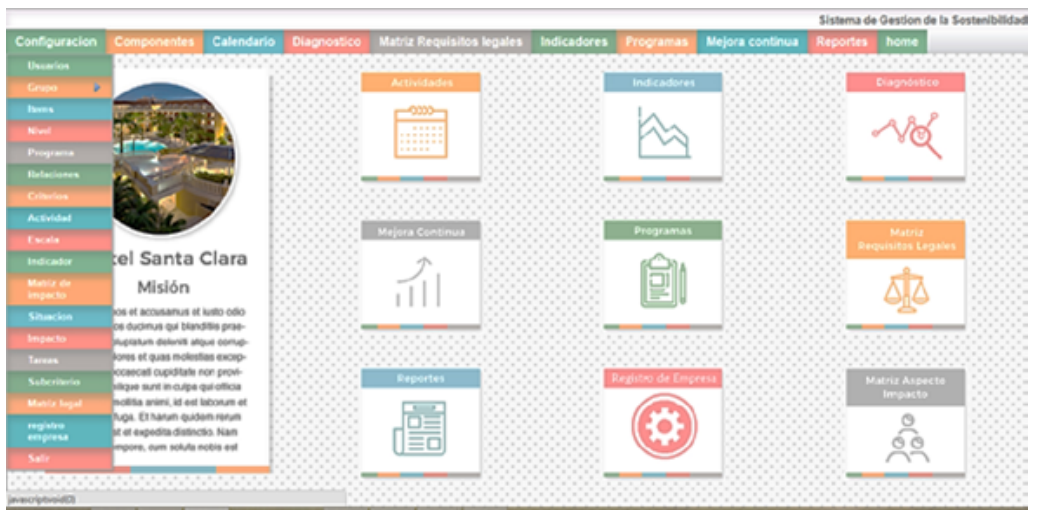

Fuente: Elaboración propia. 
a los requisitos de la norma y obtener los beneficios que esta ofrece a la organización.

\subsubsection{Módulo de diseño \\ de programas de sostenibilidad}

El módulo de diseño de programas de sostenibilidad (que aparece en la plataforma como "Programas") le permite al establecimiento el manejo de los 17 programas, divididos en las tres dimensiones: ambiental, sociocultural y económica, exigidas por la NTS TS 002: 2014. Los programas pueden ser alimentados en tiempo real por medio del software. Este módulo permite la conexión entre cada una de las áreas de la empresa a las que se hayan asignado responsabilidades. Las fechas fijadas para tal fin serán recordadas mediante un sistema de alertas que se recibirán en el correo electrónico o en los dispositivos móviles. Esta actividad automáticamente alimenta el indicador de eficacia de cada programa y evidencia aquellas actividades cuya realización está pendiente por parte de las áreas designadas.

De igual modo, las actividades referentes a cada programa se visualizan en el software mediante una agenda y/o un listado con una semaforización que representa el estado de cumplimiento de la actividad: verde, cumplimiento; amarillo, cumplimiento medio; rojo, no cumplimiento. Además, es posible subir la evidencia que da conformidad al requisito.

Este módulo de programas tiene una iteración permanente con el módulo de matrices, dado que ambos constituyen los elementos de entrada para la generación de actividades. El programa está compuesto por un objetivo medible y cuantificable, un alcance, unas actividades, unos recursos y los indicadores que permiten comprobar su eficacia.

\subsubsection{Módulo de matriz de requisitos legales}

Este módulo facilita la identificación y asignación de las normativas legales aplicables según el tipo de organización, y permite verificar si el establecimiento está cumpliendo con las normas que le corresponden de acuerdo con su razón social.

\subsubsection{Módulo de matriz de identificación de aspectos socioculturales, ambientales y económicos e impactos}

Este módulo permite identificar los aspectos e impactos ambientales, socioculturales y económicos asociados con las áreas y las actividades de la organización derivadas de la operación del establecimiento. Además, posibilita su valoración y el reconocimiento de sus acciones de control y requisito legal aplicable.

Los indicadores del software permiten visualizar el porcentaje de cumplimiento con respecto a la eficacia y efectividad de cada una de las actividades realizadas y/o asignadas para dar cumplimiento y a los programas; además, dejan ver gráficamente el porcentaje de cumplimiento de cada programa en términos generales, de modo que le permite a la gerencia la toma de decisiones en condiciones reales.

Este módulo hace posible gestionar las actividades consideradas como críticas en el sistema a partir de los resultados.

\subsubsection{Módulo de \\ mejoramiento continuo}

Por medio del módulo de mejora continua, el auditor interno del sistema de gestión para la sostenibilidad verifica si las evidencias adjuntadas en la plataforma dan cumplimiento a los requisitos de la norma, de manera que las aprueba o las rechaza. Además, permite establecer el porcentaje de eficacia y efectividad de los programas en intervalos de tiempo 
de acuerdo con la necesidad de los usuarios (mensual, trimestral, semestral, anual, entre otros). Por lo tanto, si se rechaza una actividad, para aprobar el requerimiento normativo y obtener sus beneficios, el personal encargado debe establecer acciones de mejora, definir una fecha para dar cumplimiento a tal actividad y elegir al responsable de realizarla.

\subsubsection{Módulo de indicadores}

En este módulo se encuentran las mediciones de los resultados obtenidos en cada uno de los programas de sostenibilidad implementados en la empresa, los cuales obedecen a las metas establecidas para su cumplimiento, lo cual le facilita a la dirección de la organización la toma de decisiones con base en evidencia objetiva. El cálculo se realiza automáticamente, sin que se requiera manipulación por parte del usuario, dado que los resultados se calculan con base en la información suministrada.

\subsubsection{Módulo de actividades}

Por medio de esta funcionalidad se puede observar la calendarización de las actividades que la organización tiene agendadas, así como el estado en el que se encuentra cada tarea. También se indica qué actividad está próxima a vencer, así se ayuda a la organización a mantener actualizadas y a tiempo las actividades que dan cumplimiento a la norma técnica sectorial.

\subsubsection{Módulo de reportes}

Este módulo resume toda la trazabilidad de las actividades realizadas; la organización podrá conservarla en medio impreso o magnético.

\subsubsection{Módulo de registro de empresa}

En este módulo se inscriben los datos generales de la organización, de modo que permite su identificación y configuración en la plataforma.

\section{Evaluación}

\subsection{Diseño del experimento}

La validación del software se realizó en la ciudad de Cartagena de Indias por medio de casos de prueba funcionales aplicados a un grupo focal conformado por 37 expertos entre gerentes de establecimientos de turismo, profesionales del área, trabajadores y estudiantes plenamente capacitados acerca de la temática y el manejo de la herramienta. Estos expertos fueron seleccionados entre un universo de 100 posibles individuos con las características deseadas para fingir como expertos en la validación.

El instrumento (cuestionario) evalúa las siguientes características: identidad (ítems 1 a 4), contenido (ítems 5 a 14), navegación (ítems 15 a 20), gráficas web (ítems 21 a 30), búsqueda (ítems 31 y 32), feedback (ítems 33 y 34), utilidad (ítems 35 a 38) y manual de usuario (ítems 39 a 41). Para responder los ítems se utilizó una escala de Likert de 5 categorías ordinales: "1. Muy en desacuerdo", "2. Algo en desacuerdo", "3. Ni en acuerdo ni en desacuerdo", "4. Algo de acuerdo" y "5. Muy de acuerdo".

El criterio para determinar el consenso entre las valoraciones emitidas por el grupo de evaluadores expertos fue la consistencia o concordancia existente entre sus juicios. La medida más usual para resumir el nivel de concordancia o acuerdo entre los evaluadores fueron los coeficientes de concordancia ponderados y no ponderados, AC1 de Gwet (Girota, Terwiesch y Ulrich, 2010), Alpha de Krippendorff, Kappa de Fleiss y Kappa de Conger (Kraemer, 1980).

\subsection{Instrumento de apoyo para la evaluación de NTSOFT}

Cuando a priori se diseña un instrumento o una herramienta, no existe la seguridad de 
que este/a cumpla con el objetivo para el cual fue diseñado/a. Por esta razón, dichos instrumentos o herramientas deben ser sometido/as a un riguroso proceso de validación (Carvajal, Centeno, Watson, Martínez y Sanz-Rubiales, 2011). De acuerdo con lo anterior, para comprender cómo validar las funcionalidades del sistema con el fin de definir si este apoya a las organizaciones turísticas en determinar si cumplen con los criterios de sostenibilidad según la norma técnica sectorial, se describe a continuación en qué consiste el instrumento de apoyo en la validación del software.
Los aspectos que se van a evaluar durante el proceso de validación del software se muestran en la tabla 1 .

\subsection{Evaluación del modelo acorde a la calidad, usabilidad y funcionalidad del sistema}

Para evaluar los factores anteriormente descritos y con el fin de determinar el consenso entre las evaluaciones emitidas por el grupo de evaluadores expertos, se utilizó la prueba de consistencia o concordancia entre juicios, más conocida como la técnica de coeficientes

Tabla 1. Factores que el instrumento evalúa

\begin{tabular}{|c|c|}
\hline Factores & Definición \\
\hline Identidad & $\begin{array}{l}\text { Mide los factores estéticos del software, es decir, el entorno artístico, el cual genera } \\
\text { en el usuario niveles de recordación e identidad corporativa por medio de elementos } \\
\text { gráficos y datos de la institución y del personal de contacto. }\end{array}$ \\
\hline Contenido & $\begin{array}{l}\text { Esta sección mide la incidencia de la documentación, es decir, la capacidad que ofrece } \\
\text { el sitio al proveer documentos que faciliten la interacción con el software y entre los } \\
\text { usuarios. }\end{array}$ \\
\hline Navegación & $\begin{array}{l}\text { Evalúa la facilidad de uso, es decir, el grado de satisfacción del usuario frente } \\
\text { al producto, la cual se subdivide en los siguientes subcriterios: flexibilidad de la } \\
\text { utilización de servicios como navegación, estado del sistema, menús, control de avance, } \\
\text { regreso lógico y enlaces. }\end{array}$ \\
\hline Contenido multimedia & $\begin{array}{l}\text { Se enfoca en la facilidad de comprensión, es decir, en la capacidad que ofrece el sitio } \\
\text { para usar sus recursos en tareas y condiciones de uso particular. Se subdivide en los } \\
\text { siguientes subcriterios: mapa y estructura del sitio, facilidad de uso, aplicación de } \\
\text { los requerimientos de entrada/salida, técnicas de redacción de contenido apropiado, } \\
\text { comunicación funcional, definición de perfiles de usuario. }\end{array}$ \\
\hline Búsqueda & $\begin{array}{l}\text { Evalúa la facilidad que ofrece al usuario en la consulta de recursos (filtros de búsqueda) } \\
\text { y la respuesta inmediata a la interacción del usuario con el sistema. }\end{array}$ \\
\hline Feedback & $\begin{array}{l}\text { Esta sección mide el grado de comunicación o de interacción entre el software y el } \\
\text { usuario, e indica las facilidades en cuanto a los recursos y medios que ofrece el entorno } \\
\text { para interactuar con el usuario final. }\end{array}$ \\
\hline Utilidad & $\begin{array}{l}\text { Corresponde al grado de operatividad, es decir, a la capacidad del software para cumplir } \\
\text { las funciones establecidas por el usuario. La utilidad se subdivide en los siguientes } \\
\text { subcriterios: herramientas, página inicial, enlaces y capacidad. }\end{array}$ \\
\hline Manual de usuario & $\begin{array}{l}\text { Esta sección ofrece al usuario documentación del sitio al desarrollar actividades y así } \\
\text { interactuar con este. }\end{array}$ \\
\hline
\end{tabular}

Fuente: Elaboración propia. 
C1 de Gwet y Brennan-Prediger. Se hace uso de estos coeficientes porque se tienen más de dos evaluadores, más de dos categorías para las variables y, además, una escala ordinal para las opciones de respuesta. Estos coeficientes se calculan teniendo en cuenta las distribuciones de probabilidad para las puntuaciones asignadas por los evaluadores, y se compara 1) la probabilidad de que la concordancia entre los juicios emitidos por los diferentes expertos se deba al azar con 2) la probabilidad de que esta se deba a un consenso espontáneo y objetivo en sus apreciaciones (Girota, Terwiesch y Ulrich, 2010).

Según Altman (1991), la escala más usual para establecer el nivel de concordancia es la mostrada en la tabla 2, de gran ayuda como soporte para interpretar los resultados de los factores evaluados.

La tabla 3 muestra los índices de concordancia para la validación de los diferentes factores como resultado de los criterios de los evaluadores, de acuerdo con la distribución de las frecuencias para cada nivel de la escala. Se concluye que, acorde con el índice Kappa de Gwet, la mayoría de los factores se encuentran en el nivel de "Buena", con un promedio de $87,27 \%$, así se demuestra el consenso en las respuestas de los jueces.

Tabla 2. Umbrales para los índices en la interpretación de los resultados de los análisis

\begin{tabular}{|c|c|}
\hline Valor del indice & Fuerza de concordancia \\
\hline$<0,20$ & Pobre \\
\hline $0,21-0,40$ & Débil \\
\hline $0,41-0,60$ & Moderada \\
\hline $0,61-0,80$ & Buena \\
\hline $0,81-1,00$ & Muy buena \\
\hline
\end{tabular}

Fuente: Elaboración propia.

Para cada ítem evaluado se suman y totalizan los resultados, también se presentan los intervalos del $95 \%$ de confianza y el p-valor para una prueba de hipótesis que verifica si el resultado es mayor que el $60 \%$. Con pvalores superiores a 0,05 se concluye que:

- las funcionalidades de la herramienta les son muy necesarias a las empresas del sector turismo;

Tabla 3. Índices de concordancia para la validación en todos los factores

\begin{tabular}{|l|c|c|c|c|c|c|}
\hline & Kappa de Gwet & Porcentaje & Fuerza & Brennan-Prediger & Porcentaje & Fuerza \\
\hline Identidad & 0,6019 & 88,47 & Buena & 0,5882 & 88,46 & Moderada \\
\hline Contenido & 0,7644 & 89,93 & Buena & 0,6405 & 89,93 & Buena \\
\hline Navegación & 0,6497 & 86,93 & Buena & 0,5335 & 86,94 & Moderada \\
\hline Contenido multimedia & 0,6752 & 87,99 & Buena & 0,5713 & 87,99 & Moderada \\
\hline Búsqueda & 0,6171 & 83,31 & Buena & 0,4659 & 83,31 & Moderada \\
\hline Feedback & 0,6753 & 85,52 & Buena & 0,5367 & 85,52 & Moderada \\
\hline Utilidad & 0,7845 & 90,46 & Buena & 0,6593 & 90,46 & Buena \\
\hline Manual de usuario & 0,5916 & 83,01 & Moderada & 0,4565 & 83,01 & Moderada \\
\hline
\end{tabular}

Fuente: Elaboración propia. 
- las funcionalidades de la herramienta son pertinentes para lo contemplado por la NTS TS 002:14.
La tabla 4 resume el grupo de 41 ítems evaluados. La coincidencia entre los juicios de los expertos queda evidenciada en el porcentaje de consenso de, $88,99 \%$, lo que indica un nivel bueno.

Tabla 4. Frecuencia de los factores e IC de $95 \%$ y p-valor para los ítems del constructo evaluados

\begin{tabular}{|c|c|c|c|c|c|}
\hline Factor & Ítem & Porcentaje & Intervalo confianza & $p$-valor & Significancia \\
\hline \multirow{4}{*}{ Identidad } & $\mathrm{P} 1$ & $68,42 \%$ & {$[0,5380,1,0000]$} & 0,1856 & No \\
\hline & $\mathrm{P} 2$ & $73,68 \%$ & {$[0,5926,1,0000]$} & 0,0598 & No \\
\hline & P3 & $84,21 \%$ & {$[0,7076,1,0000]$} & 0,0020 & Sí \\
\hline & P4 & $81,58 \%$ & {$[0,6780,1,0000]$} & 0,0054 & Sí \\
\hline \multirow{10}{*}{ Contenido } & P5 & $68,42 \%$ & {$[0,5380,1,0000]$} & 0,1856 & No \\
\hline & P6 & $78,95 \%$ & {$[0,6490,1,0000]$} & 0,0133 & Sí \\
\hline & P7 & $71,05 \%$ & {$[0,5651,1,0000]$} & 0,1103 & No \\
\hline & P8 & $76,32 \%$ & {$[0,6205,1,0000]$} & 0,0295 & Sí \\
\hline & P9 & $81,58 \%$ & {$[0,6780,1,0000]$} & 0,0054 & Sí \\
\hline & $\mathrm{P} 10$ & $92,11 \%$ & {$[0,8015,1,0000]$} & 0,0001 & Sí \\
\hline & P11 & $78,95 \%$ & {$[0,6490,1,0000]$} & 0,0133 & Sí \\
\hline & P12 & $84,21 \%$ & {$[0,7076,1,0000]$} & 0,0020 & Sí \\
\hline & P13 & $81,58 \%$ & {$[0,6780,1,0000]$} & 0,0054 & Sí \\
\hline & P14 & $84,21 \%$ & {$[0,7076,1,0000]$} & 0,0020 & Sí \\
\hline \multirow{6}{*}{ Navegación } & P15 & $65,79 \%$ & {$[0,5114,1,0000]$} & 0,2867 & No \\
\hline & P16 & $65,79 \%$ & {$[0,5114,1,0000]$} & 0,2867 & No \\
\hline & P17 & $68,42 \%$ & {$[0,5380,1,0000]$} & 0,1856 & No \\
\hline & P18 & $71,05 \%$ & {$[0,5651,1,0000]$} & 0,1103 & No \\
\hline & P19 & $71,05 \%$ & {$[0,5651,1,0000]$} & 0,1103 & No \\
\hline & P20 & $65,79 \%$ & {$[0,5114,1,0000]$} & 0,2867 & No \\
\hline \multirow{10}{*}{ Gráficas web } & $\mathrm{P} 21$ & $76,32 \%$ & {$[0,6205,1,0000]$} & 0,0295 & Sí \\
\hline & $\mathrm{P} 22$ & $84,21 \%$ & {$[0,7076,1,0000]$} & 0,0020 & Sí \\
\hline & $\mathrm{P} 23$ & $78,95 \%$ & {$[0,6490,1,0000]$} & 0,0133 & Sí \\
\hline & $\mathrm{P} 24$ & $65,79 \%$ & {$[0,5114,1,0000]$} & 0,2867 & No \\
\hline & $\mathrm{P} 25$ & $71,05 \%$ & {$[0,5651,1,0000]$} & 0,1103 & No \\
\hline & P26 & $42,11 \%$ & {$[0,2872,1,0000]$} & 0,9815 & No \\
\hline & $\mathrm{P} 27$ & $60,53 \%$ & {$[0,4669,1,0000]$} & 0,5000 & No \\
\hline & P28 & $65,79 \%$ & {$[0,5114,1,0000]$} & 0,2867 & No \\
\hline & P29 & $60,53 \%$ & {$[0,4669,1,0000]$} & 0,5000 & No \\
\hline & P30 & $89,47 \%$ & {$[0,7691,1,0000]$} & 0,0002 & Sí \\
\hline
\end{tabular}




\begin{tabular}{|c|c|c|c|c|c|}
\hline Factor & Ítem & Porcentaje & Intervalo confianza & $p$-valor & Significancia \\
\hline \multirow{3}{*}{ Búsqueda } & $\mathrm{P} 31$ & $84,21 \%$ & {$[0,7076,1,0000]$} & 0,0020 & Sí \\
\cline { 2 - 6 } & $\mathrm{P} 32$ & $86,84 \%$ & {$[0,7379,1,0000]$} & 0,0007 & Sí \\
\hline \multirow{4}{*}{ Feedback } & $\mathrm{P} 33$ & $86,84 \%$ & {$[0,7379,1,0000]$} & 0,0007 & Sí \\
\cline { 2 - 6 } & $\mathrm{P} 34$ & $86,84 \%$ & {$[0,7379,1,0000]$} & 0,0007 & Sí \\
\hline \multirow{5}{*}{ Utilidad } & $\mathrm{P} 35$ & $94,74 \%$ & {$[0,8352,1,0000]$} & 0,0000 & Sí \\
\cline { 2 - 6 } & $\mathrm{P} 36$ & $92,11 \%$ & {$[0,8015,1,0000]$} & 0,0000 & Sí \\
\cline { 2 - 6 } & $\mathrm{P} 37$ & $94,74 \%$ & {$[0,8352,1,0000]$} & 0,0000 & Sí \\
\cline { 2 - 6 } & $\mathrm{P} 38$ & $94,74 \%$ & {$[0,8352,1,0000]$} & 0,0000 & Sí \\
\hline \multirow{5}{*}{ Manual de usuario } & $\mathrm{P} 40$ & $84,21 \%$ & {$[0,7076,1,0000]$} & 0,0002 & Sí \\
\cline { 2 - 6 } & $\mathrm{P} 39$ & $89,47 \%$ & {$[0,7691,1,0000]$} & 0,0020 & Sí \\
\cline { 2 - 6 } & & $84,21 \%$ & {$[0,7076,1,0000]$} & & Sí \\
\hline
\end{tabular}

Fuente: Elaboración propia.

Tabla 5. Índices de concordancia para la validación en el instrumento global

\begin{tabular}{|l|c|c|c|c|}
\hline \multicolumn{1}{|c|}{ Coeficiente } & Valor & Intervalo & Porcentaje & Fuerza \\
\hline Kappa de Gwet & 0,6085 & $0,6961-0,7416$ & 88,99 & Buena \\
\hline Brennan-Prediger & 0,6068 & $0,5861-0,6276$ & 88,99 & Buena \\
\hline
\end{tabular}

Fuente: Elaboración propia.

Tabla 6. Observaciones realizadas por los expertos

\begin{tabular}{|l|l|l|}
\hline \multicolumn{1}{|c|}{ Factor } & \multicolumn{1}{|c|}{ Fuerza } & \multicolumn{1}{c|}{ Opinión de los expertos } \\
\hline Identidad & Moderada & $\begin{array}{l}\text { Los factores estéticos del software generan en el usuario niveles de recordación; } \\
\text { sin embargo, el entorno artístico - es decir, los elementos gráficos correspon- } \\
\text { dientes a la institución del cliente- podría tener un mayor protagonismo. }\end{array}$ \\
\hline Contenido & Buena & $\begin{array}{l}\text { Los jueces consideran que la estructura documental que posee el software garanti- } \\
\text { za la interacción con él y es suficiente para el cumplimiento de la NTS Ts 002:14. }\end{array}$ \\
\hline Navegación & Moderada & $\begin{array}{l}\text { Los usuarios consideran que el menú es acorde a las necesidades y que el estado } \\
\text { del sistema es adecuado, pero es necesario tener botones para el regreso lógico y } \\
\text { entre los enlaces de las funcionalidades del sistema. }\end{array}$ \\
\hline multimedia & Moderada & $\begin{array}{l}\text { La herramienta presenta facilidad para su uso, claridad en la asignación de perfi- } \\
\text { les del usuario y contenido apropiado. Sin embargo, debe mejorar el orden en que } \\
\text { se deben realizar las tareas dentro del software y la visibilidad de los reportes. }\end{array}$ \\
\hline Búsqueda & Moderada & $\begin{array}{l}\text { La herramienta ofrece al usuario facilidad de consulta. Se sugiere disminuir los } \\
\text { pasos para acceder más fácilmente al criterio de búsqueda. }\end{array}$ \\
\hline Feedback & Moderada & $\begin{array}{l}\text { El grado de interacción entre el software y el usuario es bueno. Algunas funciona- } \\
\text { lidades presentan demoras en la respuesta al usuario. }\end{array}$ \\
\hline Utilidad & $\begin{array}{l}\text { Los usuarios opinan que la herramienta es completamente pertinente y útil en el } \\
\text { sistema de gestión de sostenibilidad. }\end{array}$ \\
\hline Moderada & $\begin{array}{l}\text { Contiene todos los aspectos y conceptos necesarios, pero los usuarios consideran } \\
\text { que debe ser más iterativo. }\end{array}$ \\
\hline
\end{tabular}

Fuente: Elaboración propia. 


\section{Conclusiones}

La sostenibilidad aplicada al sector turístico es una apuesta para garantizar la continuidad de los prestadores desde los ámbitos ambiental, sociocultural y económico y para propiciar espacios para el fortalecimiento de las relaciones entre todos los actores de la cadena productiva. La NTS TS 002:2014 (sobre requisitos de sostenibilidad para establecimientos de alojamiento y hospedaje) establece una serie de compromisos que permiten garantizar la calidad turística de estos establecimientos y generar confianza entre propios y turistas.

El uso de herramientas tecnológicas asociadas con el control y seguimiento de los programas establecidos en la norma técnica sectorial es una alternativa que facilita la gestión y brinda soporte para la toma de decisiones en el nivel de la alta gerencia. El diseño del software NTSOFT para sistemas de gestión de sostenibilidad fue sometido a validación por parte de expertos y analizado por medio de los coeficientes de concordancia ponderados y no ponderados, $\mathrm{AC} 1$ de Gwet, Alpha de Krippendorff, Kappa de Fleiss y Kappa de Conger, por ser estos los métodos más apropiados para comprobar la concordancia de los resultados entre las opiniones de un conjunto de expertos y de forma objetiva determinar cuáles factores establecidos en el diseño de la herramienta son susceptibles de mejorar y cuáles han sido aprobados por los usuarios finales.

En conclusión, los índices de concordancia calculados evidencian fuerzas moderadas o buenas de consenso entre los expertos, lo cual soporta la consistencia del instrumento de validación y la consistencia y aceptabilidad de los resultados obtenidos en el análisis de frecuencias. La validación realizada deja ver que, aunque la herramienta tuvo muy buena aceptación, se deben realizar mejoras en los factores de navegación y contenido multimedia.

\section{Referencias}

Aguiar, J. G. y López, S. A. (2017). Indicadores de sostenibilidad para destinos turísticos. Medición dimensional en experiencias socio-comunitarias caso: sector Galipán, parque nacional El Ávila, estado Vargas. DELOS: Desarrollo Local Sostenible, 10(29).

Altman, D. (1991). Practical statistics for medical research. New York: Chapman \& Hall/CRC.

Arguello, C. S., Fiallos, L. R., Chinchuela, M. F., Díaz, P. B., Verdugo, C. M., Jara, C. A. y Quezada, M. I. (2017). Rentabilidad y sostenibilidad económica, ambiental y social en áreas protegidas aseguradas a través del modelo de gestión. Estudio de caso: refugios Carrel y Whymper reserva Chimborazo. European Scientific Journal, 13(2), 77-99. https://doi.org/10.19044/esj.2017. v13n2p77

Bai, X., White, D. y Sundaram, D. (18 de junio de 2013). Multi-methodological approaches in design science: A review, proposal and application. PACIS 2013 Proceedings, 159. https://core. ac.uk/download/pdf/301359603.pdf

Ballesteros, J. A., Bohórquez, C. A., Delgado, B. Y., Pérez, M. A. y Pinzón, Y. (2017). Aplicación del ciclo de mejora continua PHVA, basado en la norma técnica colombiana NTC-OHSAS 18001, al sistema de gestión en seguridad y salud en el trabajo del Hospital Local de Aguachica E. S. E., Colombia [Trabajo final de diplomado, UNAD]. Repositorio UNAD. https://repository.unad.edu. co/bitstream/handle/10596/14415/18917555. pdf? sequence $=1 \&$ isAllowed $=y$

Barquero, M. (2017). La ética en el sector del alojamiento turístico como fuente de ventaja competitiva. Revista de Comunicación de la SEECI, 21(42), 119-130. https://doi.org/10.15198/ seeci.2017.42.119-130 
Carvajal, A., Centeno, C., Watson, R., Martínez, M. y Sanz-Rubiales, A. (2011). ¿Cómo validar un instrumento de medida de la salud? Anales del Sistema Sanitario de Navarra, 34(1), 63-72. http:// scielo.isciii.es/pdf/asisna/v34n1/revision1.pdf

Catalano, B. (2013). Sostenibilidad sociocultural del turismo en el noroeste argentino. Estudio de casos: Purmamarca y Tilcara. RIAT: Revista Interamericana de Ambiente y Turismo, 9(2), 69-85. http://dx.doi.org/10.4067/riatvo19iss2pp69-85\%250718-235X

Caviedes, D. y Olaya, A. (2018). Ecoturismo en áreas protegidas de Colombia: una revisión de impactos ambientales con énfasis en las normas de sostenibilidad ambiental. Revista Luna Azul, 46, 311-330. http://dx.doi.org/10.17151/ luaz.2018.46.16

Cerezo, A. y Guevara, A. (2015). El papel estratégico de las tecnologías de la información y las comunicaciones en el turismo. International Journal of Information Systems and Software Engineering for Big Companies (IJISEBC), 2(2), 52-69. http://uajournals.com/ojs/index.php/ ijisebc/article/view/109

Congreso de Colombia. (26 de julio de 1996). Ley 300 de 1996. Por la cual se expide la Ley General de Turismo y se dictan otras disposiciones. Diario Oficial n. ${ }^{\circ}$ 42845, del 30 de julio de 1996.

(10 de julio de 2012). Por la cual se modifica la Ley 300 de 1996-Ley General de Turismo, la Ley 1101 de 2006 y se dictan otras disposiciones. Ley 158 de 2012. Diario Oficial n. ${ }^{\circ} 48.487$, del 10 de julio de 2012. http:// www.secretariasenado.gov.co/senado/basedoc/ ley_1558_2012.html

Da Rosa, F. y Silva, L. (2017). Sustentabilidade ambiental nos hotéis, contribuição teórica e metodologica. Revista Brasileira de Pesquisa en Turismo, 11(1), 39-60. http://dx.doi.org/10.7784/ rbtur.v11i1.1161
García, F., Herrera, J. y Puello, J. (2018). Ingeniería de software aplicada a una solución tecnológica orientada hacia el turismo sostenible en Cartagena de Indias (Colombia). 3c Tecnología: Glosas de Innovación Aplicadas a la Pyme, 7(1), 43-60. http://dx.doi.org/10.17993/3ctecno.2018. v7n1e25.43-60

Girota, G., Terwiesch, C. y Ulrich, K. (2010). Idea generation and the quality of the best idea. Management Science, 56(4), 591-605.

Grayson, K. y Rust, R. (2001). Interrater reliability. Journal of Consumer Psychology, 10(1), 71-73.

Instituto Colombiano de Normas Técnicas y Certificación (Icontec), MinciT y Universidad Externado de Colombia. (2014). Norma Técnica Sectorial 002:2014. Bogotá: U. Externado. https://www.mincit.gov.co/CMSPages/ GetFile.aspx?guid=f200f357-97f9-408a-a2c1$07 \mathrm{e} 1 \mathrm{a} 929 \mathrm{c} 1 \mathrm{fc}$

Kraemer, H. (1980). Extension of the kappa coefficient. Biometrics, 36(2), 207-216.

Llull Gilet, A. (2001). Contabilidad medioambiental y desarrollo sostenible en el sector turístico [Tesis doctoral, Universidad de las Islas Baleares]. Repositorio UIB. http://ibdigital.uib.es/greenstone/ collect/tesisUIB/archives/TDX-0307/105-1307. dir/TDX-0307105-130721.pdf

Martínez, L., Contreras, J., Mendoza, R. y Torres, P. (2015). INMO: A web architecture for real estate search systems. IEEE Latin America Transactions, 13(4), 1.148-1.152.

Martínez, L., Mendoza, R. y Puello, J. J. (2016). INMO: un geoportal inmobiliario aplicado a sistemas de información de búsqueda de propiedades. Revista Colombiana de Tecnologías de Avanzada, 2(28), 67-71.

Ministerio de Comercio, Industria y Turismo (MincIT). (25 de junio de 2014). Resolución 2804 
de 2014. Por la cual se reglamenta el cumplimiento de las normas técnicas de calidad expedidas por las Unidades Sectoriales de Normalización sobre Sostenibilidad Turística. Diario Oficial n. ${ }^{\circ}$ 49195, del 27 de junio de 2014, p. 15.

(19 de enero de 2015). Resolución 148. Por la cual se reglamenta el cumplimiento de las normas técnicas de calidad expedidas por las unidades sectoriales de normalización sobre sostenibilidad turística. Diario Oficial n. ${ }^{\circ} 49424$, del 13 de febrero de 2015. https://normativa.colpensiones.gov.co/colpens/docs/resolucion_mincomercioit_0148_2015.htm

Ministerio de Comercio, Industria y Turismo (MincIT) y Departamento Nacional de Planeación (DNP). (2014). Plan Sectorial de Turismo 20142018. "Turismo para la construcción de la paz". Bogotá, D. C.: MinciT y DNP. http://www.citur. gov.co/upload/publications/documentos/155. Plan_sectorial_de_turismo_2014_2018.pdf

Muñoz Gómez, L. F. (2017). Diagnóstico de los prestadores turísticos frente a la NTS-TS 003 en la Provincia del Alto Magdalena [Tesis doctoral, Universidad de Cundinamarca]. Repositorio de la Universidad de Cundinamarca. http://repositorio.ucundinamarca.edu.co/handle/20.500.12558/818

Navarrete, B. (2011). La gestión sostenible y su comunicación en el sector hotelero de la ciudad de Málaga. Prisma Social: Revista de Investigación Social, 6, 30-62. https://dialnet.unirioja.es/servlet/ articulo? codigo $=3686039$

Nunamaker, J. y Chen, M. (1990). Systems development in information systems research. En Proceedings of the Twenty- Third Annual Hawaii International Conference IEEE (pp. 631- 640). Washington, DC.: IEEE Computer Society Press.

Ochoa, F. A. y Rueda, N. R. (eds.). (2017). La implementación de las normas técnicas sectoriales en turismo sostenible en Colombia. Bogotá, D. C.: Universidad Externado de Colombia.
Pardo, C. J. (2017). Sostenibilidad y turismo en los paisajes culturales de la industrialización. Arbor, 193(785), 1-13. https://doi.org/10.3989/ arbor.2017.785n3006

Roa Rodríguez, M. I. y Sánchez Giraldo, L. R. (2018). Modelo gerencial para medir el desempeño ambiental bajo la Norma Técnica Sectorial en Turismo Sostenible en establecimientos de alojamiento y hospedaje de Usaquén en Bogotá, D. C. [Tesis de maestría, Universidad de Manizales]. Repositorio Universidad de Manizales. https://ridum.umanizales.edu.co/xmlui/handle/20.500.12746/3383

Sánchez Valdés, A. y Vargas Martínez, E. (2015). Turismo sustentable: un acercamiento a su oferta. Multiciencias, 15(3), 347-354. https://www. redalyc.org/pdf/904/90444727013.pdf

Trujillo-Casañola, Y., Febles-Estrada, A. y LeónRodríguez, G. (2014). Modelo para valorar las organizaciones al iniciar la mejora de procesos de software. Ingeniare. Revista Chilena de Ingeniería, 22(3), 412-420. http://dx.doi.org/10.4067/ S0718-33052014000300011

Unesco. (2012). Educación para el desarrollo sostenible: libro de consulta (Serie Instrumentos de Aprendizaje y Formación n. ${ }^{\circ}$ 4). París: Unesco. https://unesdoc.unesco.org/ark:/48223/ pf0000216756

unwto. (2017). Resumen del documento de análisis sobre turismo. http://www.tourism4development2017.org/wp-content/ uploads/2017/05/12522_iy2017-discussionpaper_executive-summary_es.pdf

Zúñiga Collazos, A. (2015). Impacto de la innovación en el rendimiento de las empresas turisticas en Colombia [Tesis doctoral, Universidad de Las Palmas de Gran Canaria]. Repositorio de la ULPGC. https://accedacris.ulpgc.es/bitstre am/10553/18729/4/0717200_00000_0000.pdf 